\title{
Acceso radial \\ ¿La mejor elección para la arteriografía coronaria y las intervenciones percutáneas en el síndrome coronario agudo?
}

\author{
Radial Access \\ The best choice for coronary angiography and percutaneous interventions in acute coronary \\ syndrome?
}

Ver artículo: página 209

Dra. Mónica Jaramillo Jaramillo: Medicina Interna y Cardiología, Fundación Santa $\mathrm{Fe}$ de Bogotá. Profesora Clínica Universidad de los Andes. Bogotá, D.C. (Colombia) E-mail:mjaramil@uniandes.edu.co
La elección del acceso arterial en la arteriografía coronaria en el contexto de un síndrome coronario agudo (SCA) debe ser considerada una estrategia para mejorar la eficacia y seguridad del tratamiento. Actualmente existen dos vías de acceso, la radial y la femoral, cada una de estas técnicas tiene sus ventajas y desventajas (1). La arterial radial, siendo un vaso pequeño y más superficial, tiene grandes ventajas en el momento de controlar el sangrado, particularmente después de practicar una intervención percutánea (IPC), por lo que se disminuyen las complicaciones en el sitio de acceso. Como un beneficio secundario, el acceso radial permite una deambulación rápida y, en algunos casos, mayor satisfacción del paciente. El lado oscuro del acceso radial es el tamaño de la arteria (fortaleza en el control del sangrado), tres a cuatro veces más pequeña que la femoral, lo cual limita el avance de equipos de mayor tamaño cuando éstos son necesarios. Por su tamaño la arteria radial tiende a ocluirse después del procedimiento, aunque esta complicación es generalmente asintomática puede ocurrir hasta en $10 \%$ de los casos. La curva de aprendizaje para la realización de procedimientos transradiales es mayor que para femorales. Las ventajas del acceso femoral son el mayor tamaño de la arteria, un curso predecible de catéter en la aorta ascendente, el tiempo de procedimiento y fluoroscopia un poco menor. En el caso de requerirse un balón de contrapulsación intraórtica el acceso femoral facilita su colocación (1).

Tradicionalmente el acceso femoral fue el preferido por muchos años. El acceso radial fue introducido hace más de 20 años y es usado frecuentemente en Canadá, Europa y Asia. Su uso ha aumentado progresivamente en los últimos años en Estados Unidos y de acuerdo al estudio presentado por los doctores Tamayo et al (2), publicado en este número de la revista, también está creciendo en nuestro país. Recientemente la Sociedad Europea de Cardiología publicó las guías de síndrome coronario agudo sin elevación del ST (SCASETS), donde el acceso radial para la práctica de angiografía y revascularización tiene indicación clase I con grado de recomendación A en centros experimentados en la técnica de acceso radial (3).

El estudio RIVAL(4) (The RadIal Vs FemorAL acces for coronary intervention) asignó al azar 7021 pacientes con síndrome coronario agudo, tanto infarto agudo de miocardio con elevación del ST (IMACEST) y SCASEST, a acceso radial o femoral. El resultado primario, una combinación de muerte, infarto del miocardio, ictus o sangrados mayores a los 30 días (no relacionados con revascularización miocárdica) se produjo en $3.7 \%$ de los pacientes en el grupo de acceso radial en comparación con $4 \%$ de los pacientes en el grupo de acceso femoral [HR 0.92 (IC del 95\%: 0.72, 1.17), $\mathrm{p}=0.50$ ]. Otro estudio, el SAFE-PCI (5) (Study of Access Site for Enhancement of PCI for Women) asignó al azar las mujeres sometidas a angiografía coronaria, y si se necesitaba intervención percutánea (IPC), a acceso radial o femoral. El estudio se detuvo antes de tiempo debido a una tasa de eventos mucho menor de lo esperado. Entre los 1787 pacientes incluidos (50\% presentó con SCASEST), 691 fueron sometidas a IPC. No hubo diferencia significativa en la variable principal de eficacia de sangrado o complicaciones vasculares entre el acceso radial y femoral en mujeres sometidas a IPC [radial $1.2 \%$ frente a $2.9 \%$ de la femoral; OR 0.39 (95\% CI 0.12, 1.27), $\mathrm{p}=0.12]$, mientras que en la cohorte total de mujeres sometidos a angiografía coronaria 
se detectó un beneficio [0.6\% en el grupo radial vs. $1.7 \%$ en el grupo femoral; OR 0.32 (IC 95\%: 0.12, 0.90), $\mathrm{p}=0.03$ ]. En el estudio MATRIX (Minimizing Adverse Haemorrhagic Events by TRansradial Access Site and Systemic Implementation of AngioX), 8404 pacientes con SCA se asignaron al azar a acceso radial o femoral. El acceso radial se asoció con un menor riesgo de mortalidad por cualquier causa [ $1.6 \%$ frente a $2.2 \%$; RR 0.72 (IC del 95\%: 0.53, 0.99), $\mathrm{p}=0.045$ ], mientras que las tasas de mortalidad cardiaca, infarto de miocardio y accidente cerebrovascular no fueron significativamente diferentes. Los dos grupos tuvieron tasas similares de necesidad de revascularización urgente del vaso culpable y trombosis del stent. El sangrado mayor se redujo significativamente en el grupo radial [1.6 vs. $2.3 \%$; RR 0.67 (IC de 95\%: 0.49, $0.92), p=0.013]$. Acceso radial se asoció con tasas significativamente menores de reparación quirúrgica del sitio de acceso o transfusión de productos sanguíneos. Un metaanálisis actualizado incluyendo MATRIX encontró una reducción significativa en las hemorragias mayores; muerte, infarto de miocardio o accidente cerebrovascular y en todas las causas de mortalidad asociada en el acceso radial al compararlo con el femoral.

Un punto importante de discusión es el tópico del sitio de acceso arterial y sus complicaciones, principalmente el sangrado mayor, y los eventos adversos subsiguientes. Durante una intervención percutánea el sangrado se divide en dos clases, sangrado en el sitio de acceso y sangrado diferente al del sitio de acceso. En el contexto de SCA el sangrado es más frecuente no relacionado con el sitio de acceso. En el estudio RIVAL, aproximadamente dos tercios del sangrado no estuvieron relacionados con el sitio de acceso. Los investigadores encontraron una tendencia temporal de disminución de complicaciones relacionadas con el sitio de acceso. La reducción en las complicaciones en el sitio de acceso se presentó tanto en el acceso femoral como el radial.

La disminución de sangrado en el sitio de acceso femoral, está relacionado con mejoras en la técnica y el equipo (punciones guiadas por fluoroscopia o ultrasonido), utilización de agujas de micropunción número 21 para el acceso arterial inicial, desarrollo de dispositivos para el cierre vascular al terminar el procedimiento, y la elección del agente antitrombótico en el momento de la intervención percutánea. Cada una de estas maniobras ha demostrado individualmente disminuir las complicaciones y se potencializan al usarse simultáneamente.

Los datos del beneficio del acceso radial provienen principalmente de estudios observacionales y registros de bases de datos nacionales. Al analizar el estudio RIVAL se llega a la conclusión que uno de los puntos más importantes en la presentación de complicaciones vasculares y de sangrado a nivel del sitio de acceso es la experiencia del operador. Para ingresar en este estudio se solicitó experiencia comprobada en acceso femoral y radial, lo cual condujo a tres hallazgos importantes. Primero, menor frecuencia de sangrado mayor tanto en el acceso radial como el femoral, con menores complicaciones totales en el sitio de acceso en el brazo de acceso femoral al compararlo con estudios previos. El segundo es que los operadores con alto volumen de acceso femoral tienen una reducción en eventos cardiovasculares mayores (1.3 vs $2.7 \%$, HR 0.50 IC 0.27-0.92, $\mathrm{p}=.03$ ), con una tendencia a la reducción de eventos de sangrado mayores ( $0.4 \%$ vs 1 HR 0.45 , IC $95 \% 0.16-1.3$, p =0.14) comparado con operadores sin alto volumen, lo cual sugiere que los beneficios del acceso femoral pueden estar ligados al volumen y la competencia del operador.

Por el rápido aumento en el acceso radial a nivel mundial, este acceso será usado en $50 \%$ de los procedimientos mundiales en la próxima década. Los cambios temporales muestran una disminución de la incidencia en las complicaciones vasculares y hemorrágicas relacionadas con el sitio de acceso. Esta disminución parece estar relacionada principalmente con cambios en la técnica del procedimiento y los nuevos equipos, la elección del sitio del acceso arterial desempeña un papel secundario. Se debe evaluar el riesgo de sangrado antes de una PCI, y en aquellos con alto riesgo de sangrado preferir el acceso radial.

\section{Referencias}

1. Baker NC, Ansel GM, MD, Rao SV, Jolly SS, Pichard AD, et. al. The choice of arterial access for percutaneous coronary intervention and its impact on outcome: An expert opinion perspective. Am Heart J 2015;170:13-22.

2. Tamayo N, Vallejo F, Senior JM, Fernández A, Rodríguez A, Toro JM, et al. Eficacia y seguridad del acceso radial y femoral en pacientes con sindrome coronario agudo llevados a intervencionismo coronario. REDIAL - registro de interven- 
ción por vía radial. Acta Med Colomb 2015; 40: 209-17.

3. Roffi M, Patrono C, Collet JP, Mueller C, Valgiimigli M, et al. ESC Guidelines for the management of acute coronary syndromes in patients presenting without persistent ST-segment elevation. European Heart Journal doi:10.1093/eurheartj/ ehv320.

4. Jolly SS, Yusuf S, Cairns J, et al. Radial versus femoral access for coronary angiography and intervention in patients with acute coronary syndromes (RIVAL): a randomised, parallel group, multicenter trial. Lancet 2011;377:1409-20.
5. Rao SJ, Hess CN, Barham B, Aberle LH, Anstrom CK et al. A Registry-Based Randomized Trial Comparing Radial and Femoral Approaches in Women Undergoing Percutaneous Coronary Intervention. The SAFE-PCI for Women (Study of Access Site for Enhancement of PCI for Women) Trial. J Am Coll Cardiol Intv 2014;7:857-67.

6. Valgimigli M, Gagnor A, Calabro P, Frigoli E, Leonardi S, Zaro T, et al. Radial versus femoral access in patients with acute coronary syndromes undergoing invasive management: a randomised multicentre trial. Lancet 2015;385:2465-2476. 